\title{
Zur Frage der Purpura abdominalis (Henoch).
}

\author{
Von \\ Richard Lederer, \\ Assistent der Abteilung. \\ (Aus der Kinderabteilung des k. k. Kaiser-Franz-Josef-Spitales in Wien \\ [Vorstand: Primarius Dozent Dr. Paul Moser].)
}

(Eingegangen am 6. Dezember 1912.)

Die im letzten Heft des Jahrbuches für Kinderheilkunde veröffentlichte Arbeit über Purpura abdominalis (Henoch) von Gara gibt mir Veranlassung, dieses Thema nochmals aufzunehmen und an der Hand zweier einschlägiger Fälle, die ich im Laufe des letzten Jahres zu beobachten Gelegenheit hatte, den Versuch zu machen, einige der sowohI vom Verfasser als von früheren Autoren aufgeworfenen Fragen über das Wesen dieser Erkrankung zu beantworten.

Die Klinik der Purpura abdominalis kann wohl als bekannt vorausgesetzt werden. Die beiden von Gara publizierten Fälle unterscheiden. sich im wesentlichen nicht von den bisher veröffentlichten. Der eine betraf einen 9 Jahre alten Knaben, bei dem zuerst die Bauchsymptome, Erbrechen, Koliken, Druckschmerzhaftigkeit an lokalisierter Stelle, Tenesmus und Abgang blutiger Stühle, und erst später die Hautblutungen auftraten. Der Fall ging in Heilung aus. Im zweiten Fall handelte es sich um einen 5jährigen Knaben, der schon früher Anfälle von Purpura simplex und wiederholt Nasenbluten überstanden hatte und leicht zu Hautblutungen neigte. Bei dem Kinde traten zuerst Petecchien und später Erscheinungen einer Intussusception auf, welcher das Kind auch erlag. Bei der Obduktion wurde der interessante Befund erhoben, daß von der Spitze des Invaginats ungefähr $100 \mathrm{~cm}^{3}$ Blutgerinnsel polypenartig in den tieferen Darmteil hinabhingen.

Die wesentliche Frage, um die es sich handelt, ist nun die: Ist die Purpura abdominalis primär eine hämorrhagische Diathese, die sich außer in den Gefäßen der Haut auch in denen des Darmes geltend macht und unter Umständen infolge schwerer Darmblutungen zur 
Intussusception führen kann, oder ist umgekehrt die Invagination oder ein anderer pathologischer Zustand des Magendarmkanales das Primäre und imstande, im Organismus derartige Schädigungen zu setzen, $\mathrm{da}$ auf deren Boden eine hämorrhagische Diathese entstehen kann. Schon vor Gara wurden diese beiden Standpunkte in lebhafter Weise erörtert. Die erstere Anschauung hat wohl in ganz eindeutiger Weise zuerst Carpenter vertreten, der in Darmblutungen bei bestehender hämorrhagischer Diathese die Ursache für ev. auftretende Intussusception sah, während die zweite Richtung mit allem Nachdruck Döbeli verficht. (Ich verzichte an dieser Stelle, andere Autoren zu nennen, $\mathrm{da}$ es sich im wesentlichen immer nur um diese beiden Ansichten handelt, falls überhaupt neben der Mitteilung der Kasuistik Vermutungen über das Wesen der Erkrankung geäußert werden. Ausführliche Literaturnachweise bei Döbeli, bei Dusch und Hoche, neuestens auch bei Don.) Henoch selbst, der schon in seiner ersten Zusammenfassung der Symptome zu einem einheitlichen Krankheitsbild im Jahre 1874 und auch später in seinen „Vorlesungen über Kinderkrankheiten“" eine klassische Schilderung des Leidens gibt, will die Erkrankung als hämorrhagische Diathese, aber als „Morbus sui generis“ betrachtet wissen, die streng von der Purpura simplex und dem morbus maculosus abzugrenzen sei.

Döbeli beobachtete einen Fall, bei dem schon vor der akuten Erkrankung chronische Obstipation und Abmagerung bestanden hatte, und glaubt, daß diese beiden Faktoren bestimmend gewesen seien für das Entstehen der (chronisch-rezidivierenden) Intussusception, auf deren Boden dann wieder, durch Resorption von Toxinen oder Bakterien, eine hämorrhagische Diathese entstanden sei. Er faßt seine Thesen folgendermaßen zusammen:

„1. Die Purpura abdominalis Henoch ist kein Krankheitsbild sui generis.

2. Die bei der Purpura abdominalis beobachteten Symptome vonseiten des Verdauungstractus sind der Ausdruck einer bestehenden Krankheit entweder des ganzen oder eines gewissen Abschnittes des Magendarmkanales.

3. Die Erkrankung des Magendarmkanales ist als das Primäre, die Purpura mit allen ihren Nebenerscheinungen ist als das Sekundäre aufzufassen.

4. Sämtliche Krankheiten des Verdauungskanales, welche eine Schädigung der Darmschleimhaut bewirken und dadurch die Resorption 
von Bakterien oder Toxinen ermöglichen, können bei dazu disponierten Individuen den bisher unter dem Namen Purpura abdominalis bekannten Symptomenkomplex hervorrufen.

5. Nur durch Heilung der betreffenden Krankheit des Verdauungskanals ist die Purpura und ihre Nebenerscheinungen zum Verschwinden zu bringen ${ }^{1}$ )."

Ich will nun versuchen, an Hand der beiden von mir beobachteten Fälle die aufgeworfene Frage eindeutig zu entscheiden, und reproduziere zunächst die Krankengeschichten:

Fall 1. Ludwig B., 12 Jahre alt; 10. von 12 Kindern, rechtzeitig, normal geboren. Vater gesund, Mutter an einem Frauenleiden gestorben. 4 Geschwister an unbekannter Krankheit gestorben, die übrigen gesund. Das Kind wurde I Jahr an der Brust genährt, aß seither alles mit. Jetzt ausreichende und gut zusammengesetzte gemischte Ernährüng. War immer gesund bis auf einen chronischen Bindehautkatarrh mit Blepharitis, an welchen Affektionen er auch jetzt noch leidet. Vor 1 Monat Masern ohne Komplikationen überstanden. 10 Tage vor der Aufnahme erkrankte der Junge an schmerzhaften Schwellungen einer Hand und eines Fußes. Auf Umschläge trat Besserung ein. 6 Tage später begann der Knabe mehrmals täglich zu erbrechen; es traten heftige Koliken auf, und 3 Tage später erfolgten zahlreiche Stuhlgänge (10-14 mal täglich), denen reichlich duakles Blut beigemischt war.

Bei der Aufnahme am 20. VII. 1912 fanden sich bei dem großen, kräftigen, gut genährten Jungen zahlreiche, stecknadelkopf- bis. bohnengroße, teils frische, teils ältere Petechien auf der Haut der Brust, des Rückens und der Vorderarme. Die Temperatur war unter $37^{\circ}$, der somatische Befund vollkommen normal, auch das Abdomen vollständig schmerzfrei, der Urin frei von Blut, Fiweiß und Zucker.

Therapie: Flüssige Kost, 2 mal $0,5 \mathrm{gr}$ Calcium lacticum.

23. VII. An diesem Tage erfolgte der erste Stuhlgang, in dem chemisch Blut nachgewiesen werden konnte.

24. VII. Erster Nachschub: 4 flüssige Stühle mit reichlichen Blutbeimengungen. Lebhafte Klagen über Schmerzen im Bauch, starken Tenesmus. Druckempfindlichkeit der linken Unterbauchgegend bei starker Défense musculaire daselbst. Temperatur normal. Tee, Thermophor, Calcium lacticum.

25. VII. 5 blutige Stühle. Blutbefund: 4,300 000 rote, Hämoglobin $65 \%$ (Sahli), 9400 weiße. Im gefärbten Präparat weder Poikilocytose noch Polychromasie, keine pathologischen Formen der weißen. Im Harn weder Eiweiß, Blut, noch Zucker nachweisbar.

26. VII. 5 blutige Stühle. Nooh immer starke Bauchschmerzen, lebhafter Druckschmerz in der Gegend der Flexura sigmoidea. Im Stublpräparate nach Gram neben gram-negativen Stäbchen zahlreiche gram-positive Diplo- und Streptokokken. In der Kultur keine Dysenteriebacillen, nur Bacterium coli nachweisbar.

27. VII. 4 flüssige, blutige Stühle.

29. VII. Seit gestern schmerzfrei, nur I geformter Stuhl täglich.

1) Im Original gesperrt gedruckt. 
31. VII. Zweiter Nachschub: Temperaturanstieg bis 38,3. Schmerzen im linken Knie und rechten Ellbogengelenk, die beide leicht anschwellen.

Am 1. VIII. wieder normaler Befund.

2. VIII. Dritter Nachschub: Temperatur 37,3. An beiden Unterschenkeln, besonders an der Innenseite derselben frische, stecknadelkopfgroße Peteechien. Ebensolche am Scrotum. Der linke Hode erscheint ungefähr auf die 6 fache Größe des rechten angeschwollen und bei Berührung äußerst schmerzhaft.

3. VIII. Temperatur 37,4. Blutungen und Hodenschwellung unverändert. Schwellung und Schmerzhaftigkeit der kleinen Gelenke des rechten Fußes.

4. VIII. Keine frischen Blutungen, Hoden noch immer geschwollen und schmerzhaft. Stuhl seit gestern angehalten.

6. VIII. Vierter Nachschub: Unter lebhaften Sohmerzen in der linken Fossa iliaca treten zwei reichlich BIut fübrende Entleerungen ein.

In den nächsten Tagen mäßige Schmerzen in der linken Bauchseite, die fortwährend anhalten. Stuhl nnregelmäßig, meist aussetzend, geringe Mengen Blutes enthaltend. Einmal Erbrechen.

Am 16. VIII. wixd mit dem Stuhleinea. $4 \mathrm{~cm}$ langes Stück voll kom men nekrotischen Gewebes entleert. Kein Blutabgang. Bei der mikroskopischen Untersuchung erwies sich das entleerte Gewebe als nekrotisierte Darmsohleimhaut mit angrenzenden Partien der Darmwand. Es hatte sich also offenbar um eine längerdauernde Invagination gehandelt, die durch Nekrotisierung und Abstoßung des Intussusceptums spontan ausheilte.

20. VIII. Täglich ein geformter Stuhl. Pat. beschwerdefrei.

Am 24. VIII. noch einmal Schwellung im rechten Knie, die nach 2 Tagen zurückging.

In den folgenden Tagen beschwerdefrei und ungestörte Rekonvaleszenz.

Am 6. IX. entlassen. Seither kein Nachschub (briefliche Mitteilung).

Fall 2. Hildegard F., 9 Jahre alt; das 3 . Kind von 5. Eltern und Geschwister gesund. Bis zum 12. Monat ausschließliche Brusternährung, bis zum 17. Monat Beifütterung, seit dem 19. Monat gemischte Kost. Hat früher Diphtherie und Varicellen überstanden, vor einem Jahr Pertussis und Nierenentzündung. 2 Woehen vor der Aufnahme erkrankte das Kind mit Schmerzen und Schwellungen an den Fïßßen, später in beiden Knie- und Handgelenken. An den Unterschenkeln sollen gleichzeitig rote Flecke aufgetreten sein, die später gelb wurden. An den Augen bestanden blaue Unterlaufungen. 6 Tage vor der Aufnahme traten starke Schmerzen im Bauche auf, so daß das Kind wegen Verdachtes auf Appendicitis der chirurgischen Abteilung zugewiesen wurde. Am 12. IV. wurde Pat. auf unsere Abteilung aufgenommen.

Befund bei der Aufnahme: Entsprechend großes, gracil gebautes Kind von schwacher Muskulatur und geringem Panniculus adiposus. Haut und sichtbare Schleimhäute sind blaß. An beiden Untersehenkeln und an den Streckseiten beider Ellbogengelenke stecknadelkopf- bis linsengroße Pigmentationen nach Petecchien. Am linken oberen Augenlid zwei bohnengroße, bläulich verfärbte, von grünlichgelben Rändern begrenzte Flecke (ältere Suffusionen). Sämtliche Drüsengruppen leicht geschwollen. Herz-, Lungen-, Augen-, Nase-, Ohren-, Rachen- und Nervenbefund normal. Abdomen leicht meteoristisch, nirgends druckschmerzhaft. Obstipation. Im Harn weder Eiwei $\beta_{z}$ Blut noch Zucker. 
15. IV. Erster Nachschub: Temperatur bis $38^{\circ}$. Abdomen meteoristisch gebläht. Obstipation. Klagen über Schmerzen im Bauch, Druckschmerz in der rechten Ileocoecalgegend. Weder im Harn noch im Stuhl Blut nachweisbar.

16. IV. Temperatur $38^{\circ}$, Meteorismus stärker. Kein Stuhl. 14,500 Leukocyten.

17. IV. Nachlassen der Symptome. Ein fester Stuhl.

19. IV. Zweiter Nachschub: Temperatur $38^{\circ}$. An beiden Fußrücken treten zahlreiche, frische stecknadelkopfgroße Petecohien auf. Keine Symptomo vonseiten des Verdauungstraktes.

21. IV. Dritter Nachschub: Keine frischen Blutungen. Dagegen tritt an der Haut beider Vorderarme und an den Streckseiten der Ellbogen ein Exanthem auf, wie es bisher nur als Syndrom von Purpura simplex mitgeteilt wurde. Das Exanthem besteht aus ca. linsengroßen, lebhaft roten, scharf begrenzten, in der Mitte deutlich gedellten Papeln, die später zum Teil abblaßten, zum Teil durch Bestehenbleiben bei Fingerdruck deutlich zu erkennen gaben, daß sekundär eine Blutung in die pathologisch veränderte Hautstelle stattgefunden hatte.

22. IV. Frische Nachschübe der eben beschriebenen Papeln an den Unterarmen und an den Streckseiten der Kniegelenke.

25. IV. Dieselben Papeln an den Streckseiten auch der Oberarme. Temperatur 37,2. Auf Burow-Umsohläge deutliche Besserung.

26. IV. Vollkommenes Wohlbefinden.

27. IV. Vierter Nachsohub: An beiden Fußrücken frische, stecknadelkopfgroße, etwas über die Hautoberfläche erhabene Blutungen. An beiden Kniescheiben frische Papeln and Petecchien. - Wassermann negativ.

29. IV. Fünfter Nachschub: Gestern verließ das Kind zum erstenmal für 2 Stunden das Bett. Unmittelbar nachher Temperaturanstieg bis $38,6^{\circ}$, frische Blutungen an beiden Fußrücken und Unterarmen. An beïden Kniescheiben frische Papeln. Im Urin zum erstenmal starke Blutausscheidung. Esbach 5\% $\%$. Im Sediment massenhaft granulierte, einzelne hyaline Cylinder, sehr viel weiße und rote Blutkörperchen.

2. V. Hauterscheinungen im Rückgang. Im Harn $8 \%$ Eiweiß, sehr viel Blut.

6. V. Sechster Nachschub: Unter mäBig heftigen Schmerzen in der rechten Bauchseite werden zwei flüssige, blutführende Stühle entleert. Injektion von $10 \mathrm{ccm}$ Pferdeserum.

10. V. Das Exanthem blaßt ab. Stuhl regelmäßig, mit Blut gemengt. Keine Schmerzen. Im Harn weiter Eiweiß- und Blutausscheidung.

16. V. Exanthem noch immer sichtbar. In Stuhl und Harn Blut nachweisbar.

28. V. Das Allgemeinbefinden sichtlich gebessert. Pat. nimmt an Gewicht zu, hat bessere Farbe. Im Stuhl kein Blut mehr nachweisbar. Im Harn weiter reichliche EiweiB- und Blutausscheidung. Mikroskopischer Befund unverändert. Esbach $4 \%$.

6. VI. Pat. darf zum erstenmal aufstehen und verträgt es diesmal gut. Harnbefund im gleichen. Esbach $2 \%$.

11. VI. Prophylaktisehe Diphtherieseruminjektion von $500 \mathrm{I}$. E. gelegentlich eines auf dem Saale entstandenen. Diphtheriefalles. 2 Stunden nach der Applikation fleckiges Serumexanthem an der Injektionsstelle (beschleunigte bzw. sofortige Reaktion. Das Kind hatte am 6. V. $10 \mathrm{ccm}$ Pferdeserum subcutan erhalten). 
29. VI. Das Kind hatte eine vollkommen ungestörte Rekonvaleszenz, erholte sich gut, hatte auch keine Schmerzen oder Blutungen mehr. Die Albuminurie bestand aber auch bei der Entlassung in ziemlich erheblichem Grade (Esbach $11_{2}^{1} \% 00$ ) weiter.

Es handelt sich also im ersten Fall um einen 12 jährigen Knaben, bei dem die Erkrankung in charakteristischen Schüben verlief. Dreimal traten starke Koliken mit Erbrechen, Tenesmus, zahlreichen blutigen Entleerungen auf; einmal führten dieselben zur Invagination, die spontan ausheilte, indem sich das invaginierte Stück abstieß; dreimal stellten sich Gelenkschwellungen mit rheumatoiden Schmerzen ein, einmal schwoll das Scrotum und ein Hoden an, und wiederholt traten in Schüben Blutungen in die Haut von verschiedener Größe und Intensität auf. Der zweite Fall betraf ein 9 jähriges Mädchen, das vor der Erkrankung schon einmal Nephritis mitgemacht hatte, plötzlich mit Gelenkschwellungen und Hautblutungen und wenige Tage später mit Koliken und Erbrechen erkrankte. Auch in diesem Falle zeigt die Erkrankung typischen Verlauf in Rezidiven; sowohl die Bauchsymptome als die Hautblutungen traten mehrmals auf, erstere allerdings in sehr geringer Intensität und mit nur wenigen blutigen Entleerungen. Was den Fall interessant macht, ist das Auftreten eines papulösen Exanthems an den Streckseiten der Fxtremitäten, das im ganzen viermal rezidivierte und vollkommen parallel mit dem Auftreten der übrigen Symptome, besonders der Hautblutungen in Erscheinung trat, ferner die Erkrankung an hämorrhagischer Nephritis,' die auch bei der Entlassung des Kindes noch bestand.

Fragen wir uns nun, in welchem Sinne diese beiden Fälle zu deuten sind. Bei dem Knaben, der gelegentlich seiner Erkrankung an Purpura eine Invagination akquirierte, vermissen wir in der Anamnese irgendwelche Anhaltspunkte dafür, daß schon vorher eine Erkrankung des Magendarmkanals bestanden habe, die eine Disposition zur Invagination hätte abgeben hönnen. Vielmehr begann die Erkrankung mit Gelenkschwellungen, denen erst 5 Tage später Erbrechen und Abgang blutiger Stühle folgten. Auch bei dem Mädchen ist nichts von einer Erkrankung des Magendarmkanals in früherer Zeit berichtet, und auch die Bauchsymptome während der Purpura waren nicht sehr heftig, wenn auch in deutlicher, für das von Henoch beschriebene Bild charakteristischer Weise vorhanden. Dagegen finden wir hier die Angabe, daß das Kind vor dem Auftreten der Purpura Nephritis, die in vollkommene Heilung ausgegangen war, mitgemacht habe, und tatsächlich akquirierte das 
Kind während seiner Purpura eine hämorrhagische Nephritis, die anscheinend (ich konnte später von dem Kind nichts mehr in Erfahrung bringen) in einen chronischen Verlauf überging.

Der Umstand, daß in den bisher beschriebenen Fällen Intussusception nur in ca. $50 \%$ der Erkrankungen auftrat, daß in dem von Gara selbst veröffentlichten zweiten Fall das große Blutgerinnsel im Darm einfach mechanisch zur Intussusception führen mußte, daß ferner in meinen beiden Fällen die Erkrankung mit Gelenkschwellungen und Hautblutungen begann, denen sich erst später Blutungen im Magendarmkanal hinzugesellten - alle diese Überlegungen deuten doch ungezwungen darauf hin, daß wir es bei diesen Erkrankungen mit konstitutionellen Leiden zu tun haben, die je nach verschiedener Disposition des einzelnen Individuums sich verschieden lokalisieren und äußern. Der Knabe erwies sich vor der Erkrankung scheinbar als ganz gesund. Die Erscheinungsformen der hämorrhagischen Diathese zeigten sich bei ihm weniger in der Haut als im Darm, er bekam schwere und wiederholt auftretende Darmblutungen, die schließlich zur Invagination führten. Das Mädchen zeigte nur geringfügige Bauchsymptome und ganz schwache Darmblutungen. Dagegen erwiesen sich ihre Nieren und ihre Haut, zwei Organe, deren innere Zusammengehörigkeit man ja heute mehr und mehr würdigt, als hochgradig disponiert. Das Auftreten dieses massigen papulösen Exanthems in charakteristischen Schüben und die reichlichen Hautblutungen, während die Symptome vonseiten des Verdauungstraktes in den Hintergrund traten, drängen den Gedanken an eine besondere Empfindlichkeit des Hautorganes in diesem Falle geradezu auf, und das gleichzeitige Bestehen einer in chronischen Verlauf ausgehenden hämorrhagischen Nephritis, nachdem schon früher eine akute Nierenentzündung im Anschluß an Pertussis durchgemacht worden war, erinnert stark an die débilité rénale der Franzosen. - So versehieden also diese beiden Fälle verlaufen - im großen Ganzen zeigen sie doch das bekannte Bild der Purpura abdominalis und wahren den von ihrem Entdecker so meisterhaft geschilderten Charakter. Geradeso, wie bei exsudativer Diathese bald die Haut, bald die Schleimhaut vorwiegend befallen wird, oder wie bei ,neuropathischer Diathese" die versehiedensten Manifestationen dieser Konstitutionsanomalie auftreten, wobei aber im Großen doch der bestimmte Charakter der Krankheitsanlage erkennbar bleibt, müssen wir auch für die hämorrhagische Diathese dieses Verhalten gelten lassen. Im Lichte dieser modernen Anschauungen betrachtend, werden wir also nicht fehl- 
gehen, die Purpura abdominalis Henoch als eine Form der hämorrhagischen Diathese anzusehen, die bei entsprechend disponierten Individuen bald das eine, bald das andere Symptom stärker hervortreten läßt, und werden die Intussusception als sekundäre Erscheinung auffassen.

\section{Literaturverzeichnis.}

Henoch, Berl. klin. Wochenschr. 1874, Nr. 51, S. 641.

-- Vorlesungen über Kinderkrankheiten 1899, s. $843 \mathrm{ff}$.

Dusch u. Hoche, Festschrift für Henoch 1890.

Döbeli, Schweizer Korrespondenzbl. 1908, S. 201, 240, 284.

Don, The Lancet 1909, \$. 526 .

Carpenter, British Journ. of Child. Diseases 1904, 2.

Gara, Jahrb. f. Kinderheilk. 76 (26), 5, S. 573.

Wien $\mathrm{X}$, Kundratstraße 3 . 